\title{
Sensor Fusion Algorithm by Complementary Filter for Attitude Estimation of Quadrotor with Low-cost IMU
}

\author{
A. Noordin ${ }^{1}$, M. A. M. Basri ${ }^{\star 2}$, Z. Mohamed ${ }^{3}$ \\ ${ }^{1}$ Department of Electrical Engineering Technology, Faculty of Engineering Technology, \\ Universiti Teknikal Malaysia Melaka, Hang Tuah Jaya, 76100 Durian Tunggal, Melaka, Malaysia \\ ${ }_{1,2,3}$ Department of Control and Mechatronics Engineering, Faculty of Electrical Engineering, \\ Universiti Teknologi Malaysia, 81310 Johor Bahru, Johor, Malaysia \\ ${ }^{*}$ Corresponding author, e-mail: ariffanan@fke.utm.my
}

\begin{abstract}
This paper proposes a sensor fusion algorithm by complementary filter technique for attitude estimation of quadrotor UAV using low-cost MEMS IMU. Angular rate from gyroscope tend to drift over a time while accelerometer data is commonly effected with environmental noise. Therefore, high frequency gyroscope signal and low frequency accelerometer signal is fused using complementary filter algorithm. The complementary filter scaling factor $K_{1}=0.98$ and $K_{2}=0.02$ are used to merge both gyro and accelerometer. The results show that the smooth roll, pitch and yaw attitude angle can be obtained from the low cost IMU by using proposed sensor fusion algorithm.
\end{abstract}

Keywords: MEMS, complementary filter, sensor fusion, UAV, low-cost IMU

Copyright $\odot 2018$ Universitas Ahmad Dahlan. All rights reserved.

\section{Introduction}

Nowadays, advanced in technology innovates to combine mechanical and electrical components into micro-scale object calls as Microelectromechanical systems (MEMS) [1], [2]. With MEMS technology component being inexpensive, small size, low power consumption, therefore products are redesigned to include such as an inertial measurement unit (IMU) sensor onto telecommunication, automotive industries and medical application [3-6]. In robotics, MEMS technology are the main of attitude and heading reference system (AHRS) to determine rotation, motion, location and direction (generally called attitude estimation) of mobile robots for automated navigation, autonomous underwater vehicle (AUV) for global localization systems and unmanned aerial vehicle (UAV) in aviation [1], [6-9].

However, MEMS sensor performance is commonly effected by biases and noises which tend to drift over time and reduce the accuracy of measurement. Therefore, most researcher applied sensor fusion algorithms techniques to overcome the measurement errors and obtaining accurate reading [1], [6], [7], [10].

In present-day, nonlinear filter techniques for instance extended kalman filter (EKF) [1], [11-13], unscented kalman filter (UKF) [14-17] and complementary filter (CF) [18-21] have been developed, reformed and integrated to obtain the optimal fusion of sensors or in other term called multi fusion integration (MFI) [22]-[23].

This paper focused on low cost IMU fusion using complementary filter for attitude estimation of quadrotor UAV. The system utilizes APM2.6's built in sensor (MPU6000) and external HMC5833L. Data from both sensors are converted to angles before fusion using complementary filter algorithm. The magnetic force vector from HMC5833L is used to determine yaw angle. This complementary filter is used to overcome drift on gyroscope and reduce environmental noise effect on accelerometer, and a smooth signal for attitude roll $(\phi)$ and pitch $(\theta)$ estimator is expected.

This paper organized as follows. Section 1 briefly described quadrotor UAV applications, mechanical architecture and dynamics movement, which in this research a Xconfiguration quadrotor is used. Section 2 explained in general regarding sensors used in IMU such as gyroscope, accelerometer, magnetometer and mention a sensor fusion algorithm technique based on complementary filter used in this research. Section 3 explained the 
experiments arrangement and present the outcomes, and finally, Section 4 concludes the paper by summarizing the findings.

\subsection{Quadrotor UAV}

Quadrotor is an aerial vehicle that has capabilities in vertical take-off and landing (VTOL), omni-directional flying, and easy hovering performances in limited spaces. Compare to terrain mobile robot, quadrotor can cover mission at most range due to capable of mobilizing in environment with many obstacles. Furthermore, advances in MEMS technology [24-28] and rapid prototyping technology makes quadrotor has a simplest electronics and mechanical structures design. Therefore, quadrotor always being considered in research with wide applications and various tasks such as civil commercial, calamity and meteorological observation, agricultural and military purpose such as surveillances, reconnaissance and communication [29-31] also educational purposes [32].

Quadrotor is a helicopter kind that can be controlled by varying the rotor speeds. It is an under-actuated, dynamic vehicle with four input forces and six output coordinates. To eliminate the anti-torque during manuaver, quadrotor configuration is setup as follows; 1) two diagonal motors (1 and 2) are running in the same direction, 2) the others set of motors ( 3 and 4$)$ in the other direction [33-35].

\subsection{Quadrotor Dynamics Movement}

On varying the rotor speeds altogether with the same rate, the forces will enable VTOL in altitude $z$ of the quadrotor either hovering (by increasing speeds) as Figure 1a or landing (by decreasing speeds) as Figure $1 \mathrm{~b}$. Manipulate roll angle, $\phi$ allows the quadrotor to move toward $\mathrm{y}$ direction as shown in Figure $1 \mathrm{c}$ and $1 \mathrm{~d}$. Manipulate pitch angle, $\theta$ allows the quadrotor to move toward $x$ direction as shown in Figure $1 \mathrm{e}$ and $1 \mathrm{f}$. To able the quadrotor to rotate in yaw angle $\psi$ direction, the diagonal motors need to be speed up/slow down depending on desired direction as shown in Figure $1 \mathrm{~g}$ and $1 \mathrm{~h}$ [31], [34], [33].

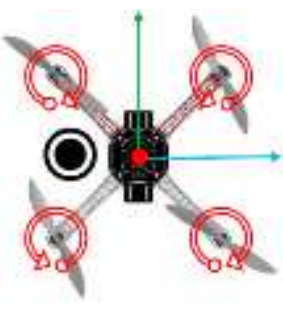

(a) Vertical up

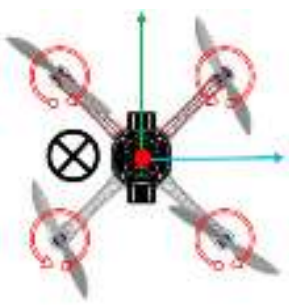

(b) Vertical down

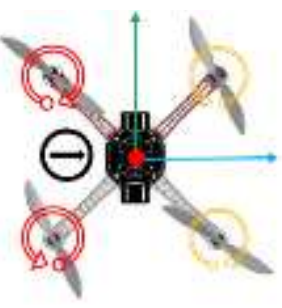

(c) Moving right

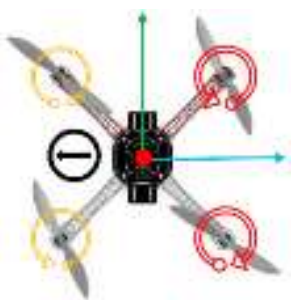

(d) Moving left

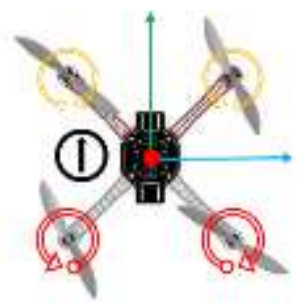

(e) Moving forward

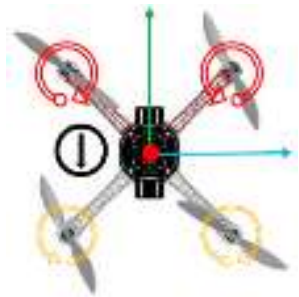

(f) Moving backward

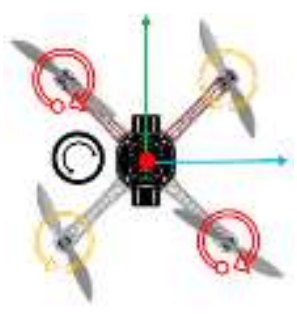

(g) Rotate right

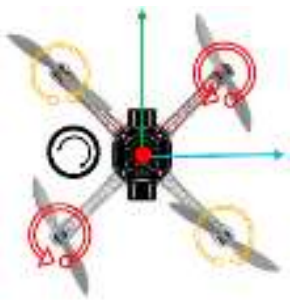

(h) Rotate left

Figure 1. Quadrotor dynamics manoeuvres

\section{Research Method}

With advanced in MEMS, combination of 3-axis gyroscopes and a 3-axis accelerometer in one chip able to provide a full 6 degree-of-freedom (DOF) motion tracking system for many applications [36]. With 3-axis digital compass additional of 3-DOF can assist on a heading for a 
system for correct orientation during locomotion. Figure 2 shows 6-DOF MPU6000 comprise of gyro and accelerometer. 3-axis compass can be attached as auxiliary sensor for attitude estimator system such used in APM2.6.
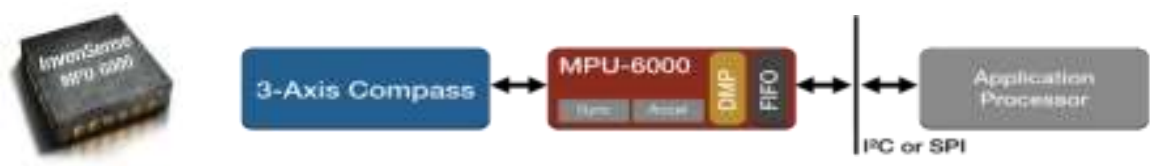

Figure 2. A 6-DOF-Gyro/Accelerometer

\subsection{Accelerometer}

An accelerometer sensor as shown in Figure 3 is a dynamic MEMS sensor used to measure acceleration forces up to three orthogonal axes. Measurement unit of accelerometer typically in gravitational motion $(\mathrm{g})$ where $1 \mathrm{~g}$ is equivalent to $9.8 \mathrm{~m} / \mathrm{s} 2$ which is acceleration cause of earth gravity. However, for linear displacement measurement, recorded data need to be integrated two times which tend to drift over a time and very sensitive to environmental noise [5],[18].

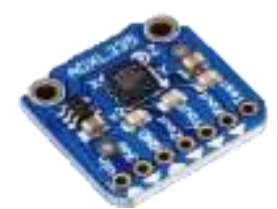

Figure 3. A 3-Axis accelerometer sensor (source: Adafruit Industries, 2017)

In other way, from an accelerometer, a tilt angle can be sensed and estimated as described on Freescale Semiconductor Application Note used by [37]. Figure 4a shows technique using two axes from an accelerometer to measure tilt angle. As shown in Figure 4b, a basic trigonometry can be applied to determine tilt angle $(\theta)$ as:

$$
\theta=\tan ^{-1}\left(\frac{A x}{A y}\right)
$$

Where magnitude of acceleration is always $1 \mathrm{~g}$ can be determined by:

$$
A=\sqrt{A_{x}^{2}+A_{y}^{2}}=1 g
$$

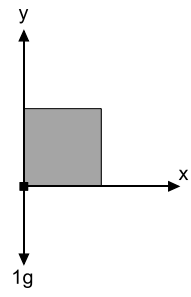

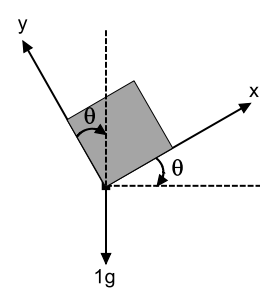

a) Tilt Angle Sensing

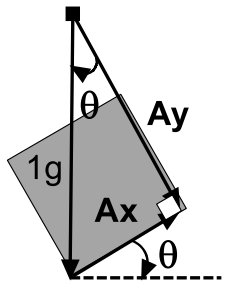

(b) Rotate about y-axis

Figure 5. Three axes tilt sensing for (a) roll angle and (b) pitch angle 


\subsection{Gyroscope}

A gyroscope is a device typically used for navigation by measurement of angular velocity and estimated changing in orientation [38]. However, for a long term, gyroscope reading tends to drift from static condition. Gyroscopes can measure rotational velocity up to three directions. In IMU, a gyroscope or gyros sensor as shown in Figure 6 is used to detect rotation speed to measure angular velocity or in other term called angular rate with measurement unit degree per second $(\% / \mathrm{s})$ or revolution per second (RPS).

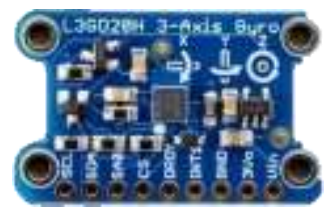

Figure 6. A 3-axis gyro sensor (Source: Adafruit Industries, 2017)

By integrating the angular rate (reading from sensor) of each axis, the roll angle $(\phi)$, the pitch angle $(\theta)$, and the yaw angle $(\psi)$ can be obtained using Equation 5 :

$$
\begin{aligned}
& \phi_{g y}=\int \omega_{x} d t \\
& \theta_{g y}=\int \omega_{y} d t \\
& \psi_{g y}=\int \omega_{z} d t
\end{aligned}
$$

Standard control range of angle in aviation system is between -180 to 180 degrees. Therefore, result from Equation 5 need to be adjusted within this range.

\subsection{Magnetometer}

A magnetometer as shown in Figure 7 can sense where the strongest magnetic force is coming from, generally used to detect magnetic north. Therefore, as for digital compass, magnetometer sensor is used to provide heading information where the heading system calculation depending to magnetic data $\left(m_{x}, m_{y}, m_{z}\right)$ therefore the compass orientation can be mathematically rotated to horizontal plane as shown in Figure 8. Based on this figure, $m_{x}, m_{y}$, and $m_{z}$ from magnetic sensor reading can be transformed to the horizontal plane $\left(X_{h}, Y_{h}\right)$ by applying the rotation as Equation 6 and Equation 7 with aided from tilt sensing roll $(\phi)$ and pitch $(\theta)$ from an accelerometer sensor. As such it is useful to determine absolute orientation in the north-east-west-south (NEWS) plane of any systems during navigation [37].

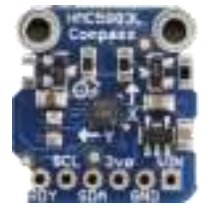

Figure 7. A 3-Axis magnetometer sensor (source: adafruit industries, 2017)

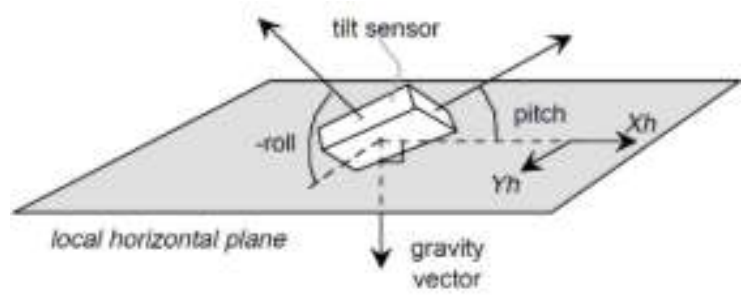

Figure 8. Tilt sensing angle in local horizontal plane defined by gravity (caruso, 2000)

$$
\begin{aligned}
& X_{h}=\mathrm{m}_{\mathrm{x}} \cos \left(\phi_{a c c}\right)+\mathrm{m}_{\mathrm{y}} \sin \left(\phi_{a c c}\right)-\mathrm{m}_{\mathrm{z}} \cos \left(\theta_{a c c}\right) \sin \left(\phi_{a c c}\right) \\
& Y_{h}=\mathrm{m}_{\mathrm{y}} \cos \left(\theta_{a c c}\right)+\mathrm{m}_{\mathrm{z}} \sin \left(\theta_{a c c}\right)
\end{aligned}
$$


From Equation 6 and Equation 7 then, the heading information can be calculated as:

$$
\text { Heading }=\psi_{h}=\tan ^{-1}\left(\frac{Y_{h}}{X_{h}}\right)
$$

\subsection{Sensor Fusion Algorithm}

There are two ways to generate attitude estimation for quadrotor; from gyroscopes by integrating it values or from accelerometer vector and magnetometer vector. Attitude estimation using gyroscope provides a good dynamics performance. While fusion using accelerometer vector and magnetometer vector give better steady-state performances [18].

In this attitude estimation, a fusion by high frequency gyroscope signal and low frequency accelerometer signal and magnetometer signal is used. This technique called complementary filter (CF) used by most researcher in [18], [20], [21]. This fusion provides reliable roll $(\phi)$ and pitch $(\theta)$ reading from gyroscope and accelerometer. While fusion tilt sensing roll $(\phi)$ and pitch $(\theta)$ from accelerometer with magnetic vector, $(m x, m y, m z)$ provide yaw $(\psi)$ which is heading direction of the system [20]. Figure 9 shows a block diagram to illustrate proposed CF technique used in this attitude estimation.

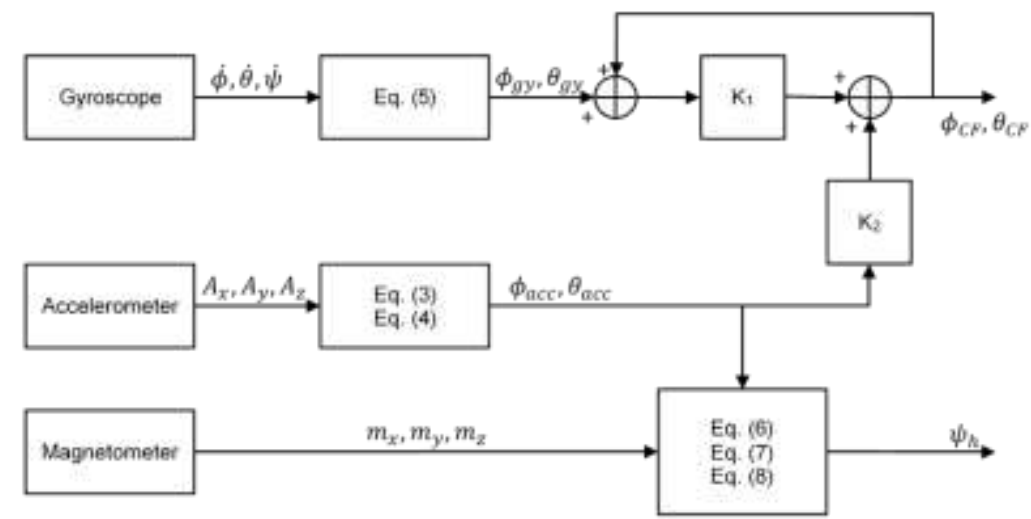

Figure 9. Complementary filter for attitude estimation

In complementary filter system, the angles will be updated with new gyroscope measurement by integral (summing) over a time and tilt sensing from accelerometer measurement with scaling factor $\mathrm{K}$. The mathematic expressions to represent complementary filter used in this study

$$
\begin{aligned}
& \phi_{C F}=K_{1}\left(\phi_{C F}+\phi_{g y}\right)+K_{2} \phi_{a c c} \\
& \theta_{C F}=K_{1}\left(\theta_{C F}+\theta_{g y}\right)+K_{2} \theta_{a c c} \\
& K_{1}+K_{2}=1
\end{aligned}
$$

\section{Results and Analysis}

Gyroscope, accelerometer, and magnetometer data from APM2.6 System is recorded and captured by serial oscilloscope. The system is program to record sensors data with sampling period of $50 \mathrm{~Hz}$ for gyroscope and accelerometer while $10 \mathrm{~Hz}$ for magnetometer. Figure 10 shows the measurement of accelerometer and gyroscope after scaling but before filtering. Plotted angle of gyroscope shows a tend to drift over a time, while accelerometer angle very sensitive to noise. In static condition both angles supposed to be at zero angle. Hence, a complementary filter is utilized to overcome the interference. 

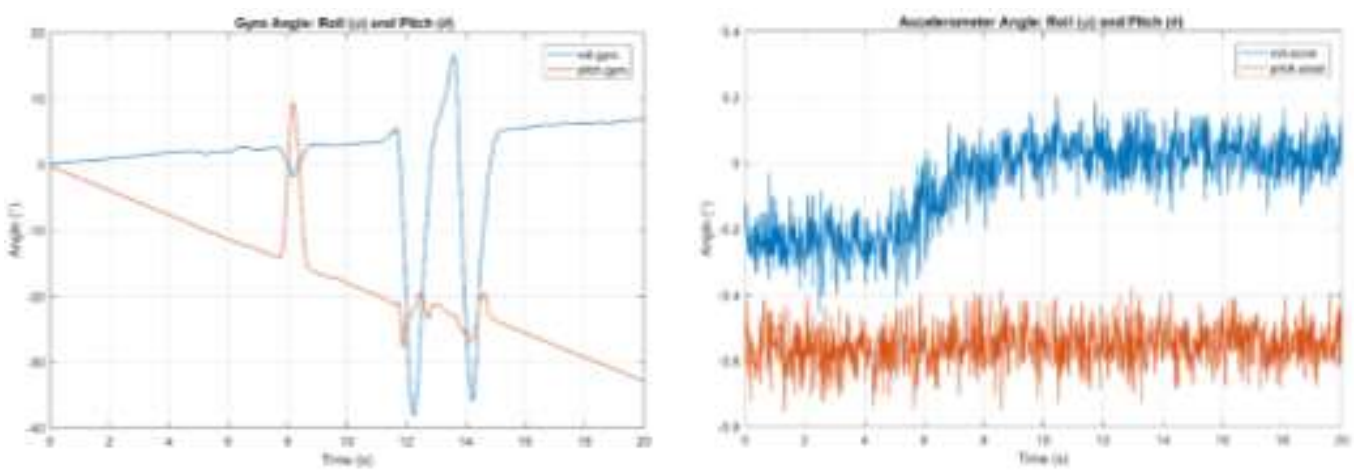

Figure 10. Gyroscope and accelerometer data converted to angle

Figure 11a and 11b show comparisons for roll angle $(\phi)$ and pitch angle $(\theta)$ respectively between gyroscope, accelerometer and after complementary filter is implemented. For Figure $11 \mathrm{a}$, the spikes at time $4 \mathrm{~s}$ and $8.4 \mathrm{~s}$ are roll angle recorded when the systems are manually rotate for positive roll angle and negative roll angle as expected the quadrotor will move to the right and left (refer to Figure $1 \mathrm{c}$ and $1 \mathrm{~d}$ respectively). While in Figure 11b, spike at $6.8 \mathrm{~s}$ and $9.2 \mathrm{~s}$ are pitch angle during quadrotor move forward and backward (refer to Figure $1 \mathrm{e}$ and $1 \mathrm{f}$ respectively). All rotation angles follow right hand rule orientation.

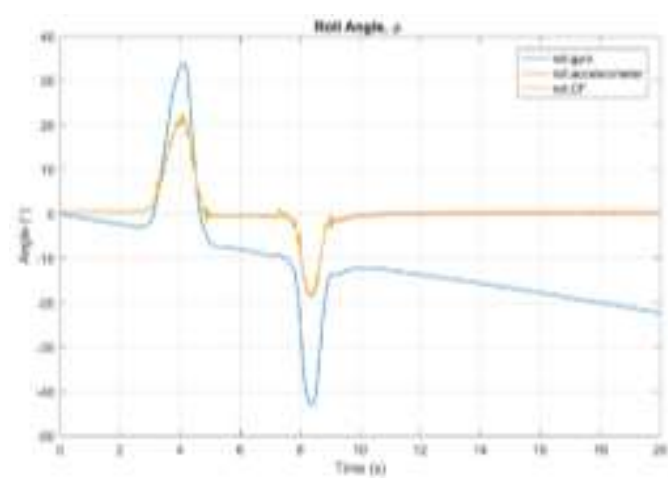

(a) roll $(\phi)$ angle

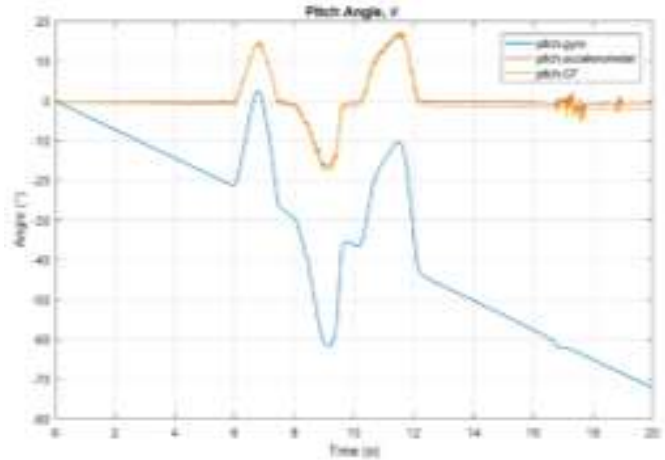

(b) pitch $(\theta)$ angle

Figure 11. Real-time roll $(\phi)$ angle measurement and pitch $(\theta)$ angle measurement

From both graphs, gyro roll $(\phi)$ and pitch $(\theta)$ signal drift over a time and not return to zero in static condition due to integral (summation) calculated from gyroscope angle reading. Accelerator roll $(\phi)$ and pitch $(\theta)$ signal from both graphs consist of noise as can be seen in Figure 10 since low cost MEMS sensor sensitive to environment. The complementary filter signal for both graph which is the estimated roll and pitch angle are the combine of gyro and accelerometer with scaling factor $K_{1}=0.98$ and $K_{2}=0.02$.

\section{Conclusion}

In this work, a complementary filter was implemented with low cost IMU to obtain attitude estimation for quadrotor system. 3-axis gyroscope and 3-axis accelerometer in MPU6000 of APM2.6 is recorded for angular rate by integrating gyros data and tilt sensing from accelerometer. Angular rate from gyros tend to drift over a time due to summing function (integral) while accelerometer is commonly effected to environmental noise. Hence a complementary filter algorithm is applied to update attitude roll $(\phi)$ and pitch $(\theta)$ by merging angular rate and tilt sensing data with appropriate scaling factor. Experiment result shows 
complementary filter technique can overcome drift problem by gyroscope which is easy to implement for quadrotor attitude estimation of low cost IMU.

\section{Acknowledgements}

The authors would like to thank Universiti Teknologi Malaysia (UTM) under the Fundamental Research Grant Scheme (R.J130000.7823.4F761), Research University Grant (Q.J130000.2523.15H39), Universiti Teknikal Malaysia Melaka (UTeM), and Ministry of Higher Education for supporting this research.

\section{References}

[1] Y Hu, Y Yan, J Liang, L Wang. A Miniature, Low-cost MEMS AHRS with Application to Posture Control of Robotic Fish. Conf. Rec.-IEEE Instrum. Meas. Technol. Conf. 2013: 1392-1395

[2] B Allotta, R Costanzi, F Fanelli. An Attitude Estimation Algorithm for Underwater Mobile Robots. IEEE/ASME Trans. Mechatronics. 2016; 21(4): 1900-1911.

[3] DR Santoso, S Maryanto, A Nadhir. Application of Single MEMS-Accelerometer to Measure 3-Axis Vibrations and 2-Axis Tilt-Angle Simultaneously. TELKOMNIKA (Telecommunication, Computing, Electronics and Control) . 2015; 13(2): 442-450.

[4] K Badshah, Q Yongyuan. Integration and Simulations of INS/GNSS System Using the Approach of Carrier Phase Measurements. Int. J. Robot. Autom. 2015; 4 (4): 243-253.

[5] U Guner, H Canbolat, A Unluturk. Design and Implementation of adaptive vibration filter for MEMS based low cost IMU. 2015 9th Int. Conf. Electr. Electron. Eng. 2015; 130-134.

[6] W Lingling, F Li, H Xioguang, Z Guofeng. Attitude Estimation for UAV with Low-Cost IMU/ADS Based on Adaptive-Gain Complementary Filter. 2016; 54: 673-680.

[7] Z Wu, Z Sun, W Zhang, Q Chen. Attitude and gyro bias estimation by the rotation of an inertial measurement unit. Meas. Sci. Technol. 2015; 26(12): 125102.

[8] DQ Duong, J Sun, TP Nguyen, L Luo. Attitude estimation by using MEMS IMU with Fuzzy Tuned Complementary Filter. Proc. 2016 IEEE Int. Conf. Electron. Inf. Commun. Technol. ICEICT 2016, no. Iceict. 2017: 372-378.

[9] BH Prasetio, AA Supianto, GE Setiawan. Earth Image Classification Design Using Unmanned Aerial Vehicle. TELKOMNIKA (Telecommunication, Computing, Electronics and Control). 2015; 13(3): 10211028.

[10] Y Wang, N Li, X Chen, M Liu. Design and implementation of an AHRS based on MEMS sensors and complementary filtering. Adv. Mech. Eng. 2014.

[11] S Sabatelli, M Galgani, L Fanucci, A Rocchi. A double-stage kalman filter for orientation tracking with an integrated processor in 9-D IMU. IEEE Trans. Instrum. Meas. 2013; 62(3): 590-598.

[12] D Veillard, F Mailly, P Fraisse. EKF-based state estimation for train localization. Proc. IEEE Sensors. 2017; 2(2): 5-7.

[13] P Yutthaa, J Tatsawan, P Chotikunnan, T Nuntachi, L Wanichsara, T Atimon. The Effect of Average Filter for Complementary Filter and Kalman Filter Based on Measurement Angle. Biomed. Eng. Int. Conf. 2016: 1-4.

[14] SA Maged, AA Abouelsoud, AMRF El Bab, T Namerikawa. Stewart Platform Manipulator: State Estimation Using Inertia Sensors and Unscented Kalman Filter. Proc-2016 3rd Int. Conf. Inf. Sci. Control Eng. ICISCE 2016: 1136-1140.

[15] WU Zhonghong, SHI Zhangsong, LIU Jian. Multi-sensor Fusion Based Unscented Attitude Estimation Method for MAVs. 2016: 5138-5142.

[16] D Yuan, X Ma, Y Liu, C Zhang. Dynamic initial alignment of the MEMS-based low-cost SINS for AUV based on unscented Kalman filter. Ocean. Shanghai, 2016.

[17] Z Lin, X Linlin, L Fanming, C Yi. Application of UKF for MEMS IMUs and fluxgate sensors based attitude and heading reference system of carriers. ICIEA 20072007 Second IEEE Conf. Ind. Electron. Appl. 2007; 0: 2278-2283.

[18] NH Ariffin, N Arsad, B Bais. Low cost MEMS gyroscope and accelerometer implementation without Kalman Filter for angle estimation. Int. Conf. Adv. Electr. Electron. Syst. Eng. 2016: 77-82.

[19] IMUW Kalman, TC Filter. Reading a IMU without Kalman: The Complementary Filter. 2013: 1-9.

[20] X Lu, J Li, W Liu. Study of Complementary Filter Algorithm for Four-rotor Helicopters Attitude Control System. 2014; 174(7): 298-306.

[21] O De Silva, GKI Mann, RG Gosine. Automated tuning of the nonlinear complementary filter for an Attitude Heading Reference observer. IEEE Work. Robot Vision, WORV. 2013: 171-176.

[22] M Faiz, B Ramli, SS Shamsudin, A Legowo. Obstacle Detection Technique Using Multi Sensor Integration for Small Unmanned Aerial Vehicle. Indonesian Journal of Electrical Engineering and Computer Science (IJEECS). 2017; 8(2): 441-449. 
[23] RC Luo, CC Chang, CC Lai. Multisensor fusion and integration: Theories, applications, and its perspectives. IEEE Sens. J. 2011; 11(12): 3122-3138.

[24] A Cahyadi, S Herdjunanto, R Hidayat. Inertial Navigation for Quadrotor Using Kalman Filter with Drift Compensation. International Journal of Electrical and Computer Engineering (IJECE). 2017; 7(5): 2596-2604.

[25] J Chang, J Cieslak, A Zolghadri, J Dávila, J Zhou. Quadrotor attitude estimation with gyroscope bias reconstruction capabilities. IFAC-PapersOnLine. 2016; 49(5): 260-265.

[26] V Kriz, P Gabrlik. UranusLink-Communication protocol for UAV with small overhead and encryption ability. in IFAC Proceedings Volumes (IFAC-PapersOnline). 2015; 48(4): 474-479.

[27] PJ Bristeau, F Callou, D Vissière, N Petit. The Navigation and Control technology inside the AR.Drone micro UAV. in IFAC Proceedings Volumes (IFAC-PapersOnline). 2011; 18(PART 1): 1477-1484.

[28] H Jafari, M Zareh, J Roshanian, A Nikkhah. An Optimal Guidance Law Applied to Quadrotor Using LQR Method. Trans. Jpn. Soc. Aeronaut. Space Sci. 2010; 53(179): 32-39.

[29] J Ajmera, V Sankaranarayanan. Point-to-point control of a quadrotor: Theory and experiment. IFACPapersOnLine. 2016; 49(1): 401-406.

[30] J Bazin, T Fields, AJ Smith. Feasibility of In-Flight Quadrotor Individual Motor Thrust Measurements. AIAA Atmos. Flight Mech. Conf., no. January 2016: 1-12.

[31] DBBVL, P Singh. A survey on design and development of an unmanned aerial vehicle (quadcopter). Int. J. Intell. Unmanned Syst. 2016; 4(2): 70-106.

[32] J Števek, M Fikar. Teaching Aids for Laboratory Experiments with AR.Drone2 Quadrotor ${ }^{\star *}$ The authors gratefully acknowledge the financial support of the Slovak Research and Development Agency under the project APVV 0551-11 and the Scientific Grant Agency of the Slovak Republic. IFACPapersOnLine. 2016; 49(6): 236-241.

[33] SK Phang, C Cai, BM Chen, TH Lee. Design and mathematical modeling of a 4-standard-propeller (4SP) quadrotor. in Proceedings of the World Congress on Intelligent Control and Automation (WCICA). 2012: 3270-3275.

[34] D Domingos, G Camargo, F Gomide. Autonomous Fuzzy Control and Navigation of Quadcopters. IFAC-PapersOnLine. 2016; 49(5): 73-78.

[35] S Bouabdallah, P Murrieri, R Siegwart, B Ghder, PR Bud. Design and Control of an Indoor Micro Quadrotor. Proc. 2004 IEEE Int. Conf. Robot. 8 Autom. New Orleans, LA April 2004: 4393-4398.

[36] S Amin, M Bin, I Reaz, SS Nasir. Integrated Vehicle Accident Detection and Location System. TELKOMNIKA (Telecommunication, Computing, Electronics and Control). 2014; 12(1): 73-78.

[37] D Derawi, J Kim. Real-time Nonlinear Complementary Observer for Low-cost Inertial Attitude System. Int. Glob. Navig. Satell. Syst. Soc. IGNSS Symp. 2013.

[38] H Fourati, N Manamanni, L Afilal, Y Handrich. Complementary observer for body segments motion capturing by inertial and magnetic sensors. IEEE/ASME Trans. Mechatronics. 2014; 19(1): 149-157. 